\title{
MÉTODOS PARA AVALIAÇÃO DA PRODUÇÃO ENXUTA: REVISÃO E ANÁLISE CRÍTICA
}

\section{METHODS FOR LEAN PRODUCTION ASSESSMENT: REVIEW AND CRITICAL ANALYSIS}

\author{
Flora Magna do Monte Vilar ${ }^{1}$; Luciano Costa Santos ${ }^{2}$; Cláudia Fabiana Gohr ${ }^{3}$; Mateus Marcelino \\ da Silva ${ }^{4}$ \\ ${ }^{1}$ Universidade Federal da Paraíba - UFPB - João Pessoa/PB - Brasil \\ floramontevilar@gmail.com \\ ${ }^{2}$ Universidade Federal da Paraíba - UFPB - João Pessoa/PB - Brasil \\ luciano@ct.ufpb.br \\ ${ }^{3}$ Universidade Federal da Paraíba - UFPB - João Pessoa/PB - Brasil \\ claudiagohr@ct.ufpb.br \\ ${ }^{4}$ Universidade Federal da Paraíba - UFPB - João Pessoa/PB - Brasil \\ mateusmarcelinos@hotmail.com
}

\begin{abstract}
Resumo
Um dos fatores críticos de sucesso para a manutenção da produção enxuta é o uso de métodos que permitam avaliar a evolução do sistema à medida que é implementado. No entanto, a produção científica sobre os métodos de avaliação ainda está numa fase inicial, carecendo de uma sistematização da literatura que permita gerar o estado da arte sobre o tema. Diante dessa necessidade, este artigo tem como principal objetivo fazer uma revisão da literatura sobre os métodos para avaliação da produção enxuta, de forma a sistematizar o conhecimento já publicado e identificar lacunas de pesquisa que possam guiar o desenvolvimento futuro do assunto. Para isso, foram adotados procedimentos de revisão sistemática para a busca e a análise dos artigos, restringindo o escopo na identificação de características úteis para o desenvolvimento de métodos de avaliação da produção enxuta. A pesquisa gerou um portfólio de 19 artigos, publicados entre os anos de 2002 e 2014. Os artigos selecionados apresentavam métodos de avaliação que foram analisados segundo quatro parâmetros: adequação ao sistema produtivo, avaliação ponderada de práticas, identificação de níveis de implementação e usabilidade. Os resultados demonstram, por um lado, que os métodos atuais apresentam procedimentos sofisticados que continuam evoluindo. Por outro lado, é possível observar que a adequação ao sistema produtivo e a avaliação sistêmica e ponderada das práticas ainda são requisitos negligenciados e que sinalizam o caminho para pesquisas futuras.
\end{abstract}

Palavras-chave: produção enxuta; avaliação; revisão de literatura.

\section{Introdução}

A Produção Enxuta teve origem do Sistema Toyota de Produção (STP) e essa expressão tornou-se conhecida pelo livro "A máquina que mudou o mundo" escrito por Womack, Jones e 
Ross (1992). Na época do estudo que gerou o livro, esses autores observaram uma superioridade significativa no desempenho das empresas japonesas em relação às empresas ocidentais, atribuindo como uma das principais causas do sucesso a busca sistemática da eliminação de desperdícios e de atividades que não agregavam valor ao produto.

A partir da divulgação de Womack, Jones e Ross (1992), cresceu o interesse da comunidade empresarial e acadêmica em torno do assunto, resultando num volume expressivo de publicações na área (JASTI; KODALI, 2015; BHAMU; SANGWAN, 2014). Dentre a vasta literatura sobre produção enxuta, destacam-se as publicações sobre a implementação da produção enxuta, que geralmente relatam fatores de influência para a implementação, métodos de implementação e métodos de avaliação da produção enxuta (MARODIN; SAURIN, 2013). As pesquisas sobre os fatores de influência para a implementação usualmente identificam fatores críticos de sucesso para a implementação, enquanto as pesquisas que apresentam métodos de implementação incorporam esses fatores de sucesso para propor métodos melhorados. No entanto, um dos fatores de sucesso na implementação da produção enxuta é o uso de métodos que permitam avaliar a evolução do sistema à medida que é implementado (NETLAND; SCHLOETZER; FERDOWS, 2015).

De acordo com Bamford et al. (2015), o processo de implementação da produção enxuta ocorre de forma interativa e pode resultar em implementações parciais. Considerando essa parcialidade na implementação, uma vez que o processo é longo e precisa de sistemas de feedback para o acompanhamento do progresso, ressalta-se a importância dos métodos que buscam avaliar o estágio de implementação da produção enxuta nas empresas. À partir da identificação do estágio de evolução de cada aspecto da produção enxuta, uma empresa passa a ter a exata noção do quanto precisa melhorar para alcançar a completa implementação do sistema enxuto.

Em função da necessidade de avaliação, surgiram alguns métodos de avaliação da produção enxuta que podem ser encontrados na literatura, como por exemplo, Vimal e Vinodh (2013) e Wan e Chen (2008). Entretanto, como o desenvolvimento dos métodos de avaliação está em uma fase inicial, pode-se considerar que o conhecimento sobre este assunto ainda se encontra muito fragmentado, carecendo de uma sistematização da literatura que permita extrair o conhecimento adequado sobre o tema.

Diante dessa demanda, este artigo tem como principal objetivo fazer uma revisão da literatura sobre os métodos para avaliação da produção enxuta, de forma a sistematizar o conhecimento já publicado e identificar lacunas de pesquisa que possam guiar o desenvolvimento futuro do assunto. Os métodos de avaliação selecionados para esta revisão de literatura foram analisados segundo quatro parâmetros: adequação ao sistema produtivo, avaliação ponderada de práticas, identificação de níveis de implementação e usabilidade. Esses parâmetros foram determinados como requisitos para um método de avaliação, de maneira que a análise crítica das 
publicações selecionadas pudesse fornecer informações úteis para o desenvolvimento de novos métodos de avaliação da produção enxuta.

Este artigo complementa as pesquisas de Camacho-Miñano, Moyano-Fuentes e SacristánDíaz (2013) e Walter e Tubino (2013), que também apresentaram revisões de literatura sobre avaliação da produção enxuta. Além de apresentar uma atualização da bibliografia, este artigo tem um enfoque diferente dos artigos citados. Por um lado, Camacho-Miñano, Moyano-Fuentes e Sacristán-Díaz (2013) fazem um estudo focado na avaliação dos resultados da produção enxuta em termos de desempenho e não em função do grau de implementação de suas práticas. Por outro lado, Walter e Tubino (2013) estudaram os métodos de avaliação do grau de implementação da produção enxuta, tal como faz este estudo. Porém, Walter e Tubino (2013) analisaram parâmetros diferentes dos propostos neste trabalho, possuindo um caráter mais genérico e menos direcionado para o desenvolvimento de métodos de avaliação. Por ter um escopo mais restrito, este estudo selecionou uma quantidade de artigos menor que os estudos anteriores, porém mais focado na identificação de características úteis para os métodos de avaliação da produção enxuta.

Após esta introdução, a estrutura do artigo segue com os procedimentos metodológicos utilizados para o desenvolvimento da pesquisa. Na seção seguinte é apresentada uma visão geral das pesquisas selecionadas para a revisão. Em seguida, descreve-se a contribuição dos métodos de avaliação da implementação da produção enxuta. Posteriormente, são apresentadas as lacunas da literatura e as perspectivas para futuras pesquisas, à partir da análise realizada. $\mathrm{O}$ artigo é finalizado com as conclusões que sintetizam o trabalho.

\section{Procedimentos metodológicos}

De acordo com Webster e Watson (2002), o desenvolvimento de uma revisão da literatura é essencial para avançar no conhecimento de um determinado assunto, pois auxilia no desenvolvimento de teorias cujas temáticas são consideradas incipientes. Considerando o estágio incipiente das pesquisas sobre a avaliação da produção enxuta, a pesquisa apresentada neste artigo se propôs a analisar como a avaliação da implementação da produção enxuta vem sendo tratada pelas pesquisas internacionais publicadas recentemente, abordando especificamente os métodos de avaliação.

O método de revisão da literatura selecionado para o desenvolvimento desta pesquisa foi a revisão sistemática, que se utiliza de métodos rigorosos e explícitos de pesquisa para identificar, selecionar e avaliar a qualidade do material; assim como analisar e descrever as reais contribuições para o desenvolvimento da pesquisa (DE-LA-TORRE-UGARTE-GUANILO; TAKAHASHI; BERTOLOZZI, 2011; CORDEIRO et al., 2007). Assim, a revisão sistemática da literatura se 
diferencia da revisão da literatura narrativa (ou tradicional), pois se utiliza de métodos bem detalhados de pesquisa, respondendo a uma pergunta pontual (DE-LA-TORRE-UGARTEGUANILO; TAKAHASHI; BERTOLOZZI, 2011; CORDEIRO et al., 2007).

A estrutura metodológica adotada para o levantamento bibliográfico é apresentada na Figura 1. O levantamento bibliográfico foi realizado em bases de dados que possuem relevância no meio acadêmico, proporcionando assim maior confiabilidade à pesquisa. As bases de dados selecionadas foram as seguintes: Portal de Periódicos da Capes, Web of Science e EBSCO. A pesquisa foi restrita aos artigos que possuíam as seguintes palavras-chave em seu título: Leanness, Leanness Assessment e Leanness Evaluation. Além disso, a busca foi limitada a artigos publicados entre os anos de 1997 e 2014. A data inicial foi escolhida com a finalidade de verificar o efeito gerado pelo artigo pioneiro de Karlsson e Åhlström (1996), que se tornou um clássico da literatura sobre avaliação da produção enxuta.

Figura 1 - Estrutura metodológica da pesquisa

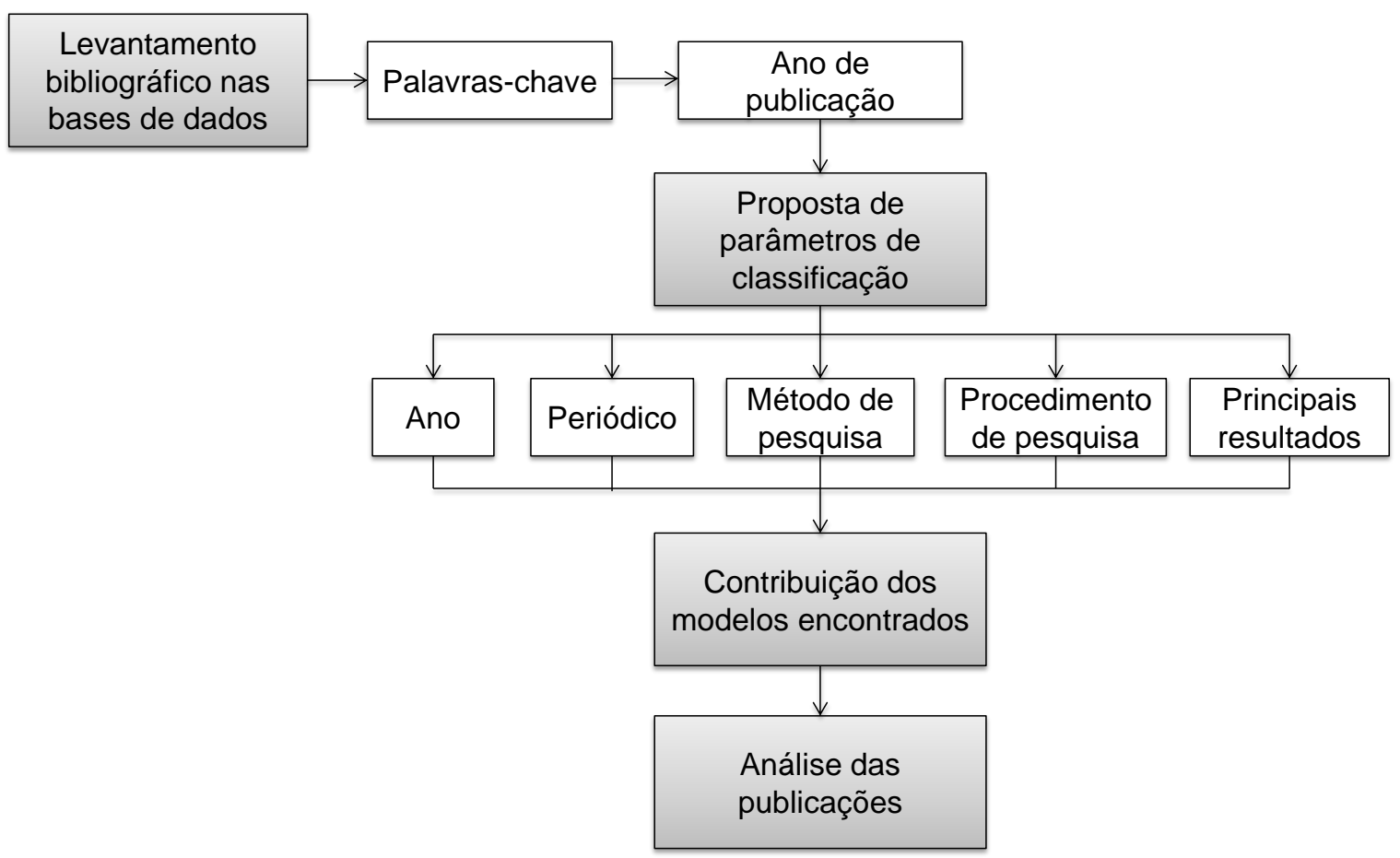

Fonte: Elaborada pelos autores.

$\mathrm{Na}$ busca realizada foram selecionados apenas os artigos diretamente relacionados com o tema produção enxuta. Com isso, foram obtidas as seguintes quantidades de acordo com a base de dados e palavras-chave, como é mostrado na Tabela 1.

Posteriormente foram analisados os títulos, os resumos e as palavras-chave dos artigos encontrados, selecionando apenas aqueles que possuíam como foco a avaliação da produção enxuta. Com isso, foram encontrados os seguintes resultados, ilustrados na Tabela 2. 
Tabela 1 - Quantidade de artigos encontrados

\begin{tabular}{cccc}
\cline { 2 - 4 } & & Bases de dados & \\
\hline Palavras-chave & Periódicos Capes & Web of Science & EBSCO \\
\hline Leanness & 140 & 93 & 2 \\
Leanness Assessment & 77 & 5 & 1 \\
Leanness Evaluation & 5 & 4 & 19 \\
\hline Total & 222 & 102 & \\
\hline
\end{tabular}

Fonte: Elaborada pelos autores.

Tabela 2 - Quantidade de artigos "filtrados"

\begin{tabular}{cccc}
\cline { 2 - 4 } & & Bases de dados & \\
\hline Palavras-chave & Periódicos Capes & Web of Science & EBSCO \\
\hline Leanness & 16 & 10 & 3 \\
Leanness Assessment & 9 & 3 & 0 \\
Leanness Evaluation & 3 & 3 & 4 \\
\hline Total & 28 & 16 & 1 \\
\hline
\end{tabular}

Fonte: Elaborada pelos autores.

A Tabela 2 apresenta uma diminuição considerável na quantidade de artigos que abordam como assunto principal a avaliação do grau de implementação da produção enxuta. Posteriormente a esse processo de "filtragem", foram desconsiderados os artigos que foram encontrados em mais de uma base de dados, ou seja, artigos repetidos, resultando em um total de 19 artigos selecionados para o portfólio a ser analisado.

A classificação dos artigos selecionados se deu de acordo com suas características, obedecendo aos seguintes parâmetros: ano de publicação, periódico no qual foi publicado, métodos de pesquisa, procedimentos de pesquisa e principais resultados. Os parâmetros foram selecionados com o objetivo de sintetizar as principais características das publicações recentes sobre o tema, assim como apresentar uma perspectiva geral de cada método analisado.

Com relação aos métodos de pesquisa os artigos foram classificados como pesquisa quantitativa ou pesquisa qualitativa. Como pesquisa quantitativa classificaram-se aqueles artigos que possuíam mensuração de variáveis em seu escopo, já como pesquisa qualitativa foram classificados aqueles que buscaram obter e interpretar informações sobre os indivíduos e sobre o ambiente que compõe a pesquisa (MARTINS, 2010).

Os artigos também foram classificados quanto aos procedimentos de pesquisa utilizados, sendo eles: estudo de caso, pesquisa-ação, survey, modelagem e simulação. Segundo Martins (2010), no estudo de caso o envolvimento do pesquisador com a organização ocorre apenas nas visitas realizadas para a coleta de dados, já na pesquisa-ação o envolvimento do pesquisador é maior, pois ele faz parte das mudanças organizacionais ocorridas provenientes da pesquisa. Ainda de acordo com o mesmo autor, no survey o pesquisador pode ou não ter proximidade com o objeto 
de estudo, já que em geral o instrumento de coleta de dados é o questionário. Para Martins (2010), tanto na modelagem quanto na simulação o pesquisador é capaz de manipular as variáveis, em sistemas que procuram representar a realidade.

A classificação dos artigos foi útil para que se obtivesse uma visão panorâmica das publicações. Após a classificação dos artigos, a pesquisa seguiu com a análise do conteúdo dos mesmos, com o objetivo de identificar as contribuições científicas já realizadas e as lacunas deixadas para a realização de pesquisas futuras. As lacunas foram identificadas com base no atendimento aos requisitos para um método de avaliação (adequação ao sistema produtivo, avaliação ponderada de práticas, identificação de níveis de implementação e usabilidade), cuja necessidade será explicada posteriormente.

\section{Visão panorâmica das publicações}

Após a leitura dos artigos selecionados, foi possível coletar as informações necessárias para a classificação dos mesmos conforme os parâmetros propostos anteriormente (Quadro 1). Este quadro apresenta o título dos artigos, os autores, seguido do ano de publicação, periódico no qual foi publicado, abordagem e método de pesquisa e os principais resultados de cada artigo analisado.

Quadro 1 - Classificação dos métodos de avaliação da implementação da produção enxuta

\begin{tabular}{|c|c|c|c|c|c|c|}
\hline Título & Autores & Ano & Periódico & Abordagem & Método & Principais resultados \\
\hline $\begin{array}{c}\text { A model for } \\
\text { evaluating the } \\
\text { degree of leanness of } \\
\text { manufacturing firms }\end{array}$ & $\begin{array}{l}\text { Soriano- } \\
\text { Meier e } \\
\text { Forrester }\end{array}$ & 2002 & $\begin{array}{l}\text { Integrated } \\
\text { Manufacturing } \\
\text { Systems }\end{array}$ & $\begin{array}{l}\text { Qualitativa e } \\
\text { Quantitativa }\end{array}$ & Survey & $\begin{array}{l}\text { Avalia o grau de adoção } \\
\text { dos princípios da produção } \\
\text { enxuta e o compromisso da } \\
\text { gestão com a mesma por } \\
\text { meio de um questionário. }\end{array}$ \\
\hline $\begin{array}{l}\text { Applying lean } \\
\text { assessment tools in } \\
\text { Chinese hi-tech } \\
\text { industries }\end{array}$ & Taj & 2005 & $\begin{array}{c}\text { Management } \\
\text { Decision }\end{array}$ & $\begin{array}{l}\text { Qualitativa e } \\
\text { Quantitativa }\end{array}$ & Survey & $\begin{array}{l}\text { Avalia nove principais } \\
\text { áreas da produção em } \\
\text { fábricas na China por meio } \\
\text { de um questionário. Os } \\
\text { resultados são exibidos em } \\
\text { uma planilha eletrônica e é } \\
\text { fornecido um gráfico de } \\
\text { perfil enxuto para ilustrar a } \\
\text { situação atual da planta e as } \\
\text { oportunidades de melhoria. }\end{array}$ \\
\hline $\begin{array}{c}\text { Application of } \\
\text { Mahalanobis } \\
\text { distance as a lean } \\
\text { assessment metric }\end{array}$ & $\begin{array}{l}\text { Srinivasara- } \\
\text { ghavan e } \\
\text { Allada }\end{array}$ & 2006 & $\begin{array}{l}\text { International } \\
\text { Journal of } \\
\text { Advanced } \\
\text { Manufacturing } \\
\text { Technology }\end{array}$ & Quantitativa & Survey & $\begin{array}{c}\text { Fornece uma medida } \\
\text { quantitativa de avaliação da } \\
\text { produção enxuta utilizando } \\
\text { a distância de Mahalanobis. } \\
\text { Ao final permite que a } \\
\text { empresa identifique as } \\
\text { melhorias e a direção das } \\
\text { mesmas de acordo com o } \\
\text { capital da empresa. }\end{array}$ \\
\hline
\end{tabular}




\begin{tabular}{|c|c|c|c|c|c|c|}
\hline Título & Autores & Ano & Periódico & Abordagem & Método & Principais resultados \\
\hline $\begin{array}{l}\text { Measuring the } \\
\text { leanness of } \\
\text { manufacturing } \\
\text { system: a case study } \\
\text { of Ford Motor } \\
\text { Company and } \\
\text { General Motors }\end{array}$ & $\begin{array}{l}\text { Bayou e } \\
\text { Korvin }\end{array}$ & 2008 & $\begin{array}{c}\text { Journal of } \\
\text { Engineering and } \\
\text { Technology } \\
\text { Management }\end{array}$ & Quantitativa & $\begin{array}{l}\text { Estudo de } \\
\text { Caso }\end{array}$ & $\begin{array}{l}\text { Usando uma metodologia } \\
\text { de lógica fuzzy, o estudo de } \\
\text { caso apresenta os passos de } \\
\text { um algoritmo e aplica-o a } \\
\text { uma indústria } \\
\text { automobilística. }\end{array}$ \\
\hline $\begin{array}{c}\text { Lean manufacturing } \\
\text { performance in } \\
\text { China: assessment of } \\
65 \text { manufacturing } \\
\text { plants }\end{array}$ & Taj & 2008 & $\begin{array}{l}\text { Journal of } \\
\text { Manufacturing } \\
\text { Technology } \\
\text { Management }\end{array}$ & $\begin{array}{l}\text { Qualitativa e } \\
\text { Quantitativa }\end{array}$ & Survey & $\begin{array}{l}\text { Avalia } 65 \text { fábricas na China } \\
\text { por meio de um } \\
\text { questionário que avalia a } \\
\text { produção enxuta por meio } \\
\text { de } 40 \text { questões dirigidas a } \\
\text { gerentes de manufatura. }\end{array}$ \\
\hline $\begin{array}{l}\text { A leanness measure } \\
\text { of manufacturing } \\
\text { systems for } \\
\text { quantifying impacts } \\
\text { of lean initiatives }\end{array}$ & Wan e Chen & 2008 & $\begin{array}{l}\text { International } \\
\text { Journal of } \\
\text { Production } \\
\text { Research }\end{array}$ & Quantitativa & $\begin{array}{l}\text { Modelagem } \\
\text { e Simulação }\end{array}$ & $\begin{array}{l}\text { Propôs o DEA (data } \\
\text { envelopment analysis) } \\
\text { como metodologia para } \\
\text { medir o grau de } \\
\text { enxugamento (leanness) de } \\
\text { sistemas de manufatura. }\end{array}$ \\
\hline $\begin{array}{l}\text { Decision support for } \\
\text { lean practitioners: } a \\
\text { web-based adaptive } \\
\text { assessment approach }\end{array}$ & Wan e Chen & 2009 & $\begin{array}{l}\text { Computers in } \\
\text { Industry }\end{array}$ & Quantitativa & $\begin{array}{l}\text { Modelagem } \\
\text { e Simulação }\end{array}$ & $\begin{array}{l}\text { Os autores desenvolvem } \\
\text { uma ferramenta de apoio à } \\
\text { decisão para orientar as } \\
\text { empresas no processo de } \\
\text { implementação da produção } \\
\text { enxuta. O software avalia o } \\
\text { estado atual da empresa, } \\
\text { identifica os pontos de } \\
\text { melhoria e sugere as } \\
\text { ferramentas e técnicas que } \\
\text { devem ser utilizadas para os } \\
\text { planos de ação das } \\
\text { melhorias. }\end{array}$ \\
\hline $\begin{array}{c}\text { Development of } \\
\text { index for measuring } \\
\text { leanness: study of an } \\
\text { Indian auto } \\
\text { component industry }\end{array}$ & $\begin{array}{l}\text { Singh, Garg } \\
\text { e Sharma }\end{array}$ & 2010 & $\begin{array}{l}\text { Measuring } \\
\text { Business } \\
\text { Excellence }\end{array}$ & Quantitativa & $\begin{array}{l}\text { Estudo de } \\
\text { Caso }\end{array}$ & $\begin{array}{l}\text { Utilizando a lógica fuzzy } \\
\text { foram desenvolvidos } \\
\text { índices enxutos e } \\
\text { apresentados } \\
\text { separadamente numa escala } \\
\text { de } 100 \text { pontos. }\end{array}$ \\
\hline $\begin{array}{l}\text { Measuring the } \\
\text { leanness of an } \\
\text { organisation }\end{array}$ & Bhasin & 2011 & $\begin{array}{l}\text { International } \\
\text { Journal of Lean } \\
\text { Six Sigma }\end{array}$ & Quantitativa & $\begin{array}{l}\text { Estudo de } \\
\text { Caso }\end{array}$ & $\begin{array}{l}\text { Classifica a organização em } \\
\text { sete fases de acordo com } \\
\text { uma pontuação fornecida } \\
\text { pelo modelo proposto que é } \\
\text { composto por } 104 \text { índices } \\
\text { agrupados em } 12 \\
\text { categorias. }\end{array}$ \\
\hline $\begin{array}{l}\text { Extracting leanness } \\
\text { criteria by } \\
\text { employing the } \\
\text { concept of Balanced } \\
\text { Scorecard }\end{array}$ & $\begin{array}{l}\text { Seyedhossei- } \\
\text { ni } \text { et al. }\end{array}$ & 2011 & $\begin{array}{c}\text { Expert Systems } \\
\text { with } \\
\text { Applications }\end{array}$ & Quantitativa & $\begin{array}{l}\text { Estudo de } \\
\text { Caso }\end{array}$ & $\begin{array}{c}\text { Foi introduzido um método } \\
\text { sistemático e lógico para } \\
\text { extrair e definir critérios } \\
\text { para uma empresa ser } \\
\text { enxuta usando o conceito } \\
\text { do Balanced Scorecard. } \\
\text { Este método foi combinado } \\
\text { com outros métodos de } \\
\text { decisão, como o Delphi e } \\
\text { DEMATEL. }\end{array}$ \\
\hline
\end{tabular}




\begin{tabular}{|c|c|c|c|c|c|c|}
\hline Título & Autores & Ano & Periódico & Abordagem & Método & Principais resultados \\
\hline $\begin{array}{c}\text { Evaluation of } \\
\text { leanness using fuzzy } \\
\text { association rules } \\
\text { mining }\end{array}$ & $\begin{array}{l}\text { Vinodh, } \\
\text { Prakash e } \\
\text { Selvan }\end{array}$ & 2011 & $\begin{array}{l}\text { International } \\
\text { Journal of } \\
\text { Advanced } \\
\text { Manufacturing } \\
\text { Technology }\end{array}$ & Quantitativa & $\begin{array}{l}\text { Estudo de } \\
\text { Caso }\end{array}$ & $\begin{array}{l}\text { O método utiliza atributos } \\
\text { para avaliar a produção } \\
\text { enxuta que foram baseados } \\
\text { em unidades de decisão } \\
\text { quantitativa e qualitativa. } \\
\text { Esses atributos representam } \\
\text { as medidas de desempenho } \\
\text { da produção enxuta. Os } \\
\text { autores utilizam a lógica } \\
\text { fuzzy associada à técnica de } \\
\text { mineração de dados FARM. }\end{array}$ \\
\hline $\begin{array}{l}\text { Leanness assessment } \\
\text { using multigrade } \\
\text { fuzzy approach }\end{array}$ & $\begin{array}{l}\text { Vinodh e } \\
\text { Chintha }\end{array}$ & 2011 & $\begin{array}{l}\text { International } \\
\text { Journal of } \\
\text { Production } \\
\text { Research }\end{array}$ & Quantitativa & $\begin{array}{l}\text { Estudo de } \\
\text { Caso }\end{array}$ & $\begin{array}{l}\text { Os autores apresentam um } \\
\text { modelo conceitual } \\
\text { composto por cinco } \\
\text { facilitadores enxutos, } 20 \\
\text { critérios enxutos e vários } \\
\text { atributos. Foram escolhidos } \\
\text { cinco especialistas em } \\
\text { produção enxuta para a } \\
\text { avaliação. Posteriormente } \\
\text { foi aplicada a lógica fuzzy e } \\
\text { a partir das notas fornecidas } \\
\text { pelos especialistas foi } \\
\text { gerado o grau de } \\
\text { enxugamento da empresa. }\end{array}$ \\
\hline $\begin{array}{l}\text { Thirty criteria based } \\
\text { leanness assessment } \\
\text { using fuzzy logic } \\
\text { approach }\end{array}$ & $\begin{array}{l}\text { Vinodh e } \\
\text { Vimal }\end{array}$ & 2012 & $\begin{array}{l}\text { International } \\
\text { Journal of } \\
\text { Advanced } \\
\text { Manufacturing } \\
\text { Technology }\end{array}$ & Quantitativa & $\begin{array}{l}\text { Estudo de } \\
\text { Caso }\end{array}$ & $\begin{array}{l}\text { Apresenta como resultado } \\
\text { um modelo conceitual de } \\
\text { avaliaçãa do grau de } \\
\text { enxugamento com } 30 \\
\text { critérios e identifica os } \\
\text { principais obstáculos da } \\
\text { implementação. }\end{array}$ \\
\hline $\begin{array}{l}\text { Development of } \\
\text { computerized } \\
\text { decision support } \\
\text { system for leanness } \\
\text { assessment using } \\
\text { multi grade fuzzy } \\
\text { approach }\end{array}$ & $\begin{array}{l}\text { Vinodh e } \\
\text { Kumar }\end{array}$ & 2012 & $\begin{array}{c}\text { Journal of } \\
\text { Manufacturing } \\
\text { Technology } \\
\text { Management }\end{array}$ & Quantitativa & $\begin{array}{l}\text { Estudo de } \\
\text { Caso }\end{array}$ & $\begin{array}{c}\text { Desenvolveu um sistema de } \\
\text { apoio à decisão (DSS) para } \\
\text { a avaliação do grau de } \\
\text { enxugamento pela lógica } \\
\text { fuzzy (MGFLA) (DSS- } \\
\text { MGFLA). Além de permitir } \\
\text { a avaliação precisa da } \\
\text { produção enxuta, o sistema } \\
\text { também permite a } \\
\text { identificação de áreas de } \\
\text { melhoria. }\end{array}$ \\
\hline $\begin{array}{l}\text { Leanness evaluation } \\
\text { using IF-THEN rules }\end{array}$ & $\begin{array}{l}\text { Vimal e } \\
\text { Vinodh }\end{array}$ & 2012 & $\begin{array}{l}\text { International } \\
\text { Journal of } \\
\text { Advanced } \\
\text { Manufacturing } \\
\text { Technology }\end{array}$ & Quantitativa & $\begin{array}{l}\text { Estudo de } \\
\text { Caso }\end{array}$ & $\begin{array}{l}\text { O estudo contribui para o } \\
\text { desenvolvimento de um } \\
\text { modelo abrangente de } \\
\text { avaliação do grau de } \\
\text { enxugamento, usando a } \\
\text { lógica fuzzy baseada no } \\
\text { mecanismo IF-THEN. }\end{array}$ \\
\hline
\end{tabular}




\begin{tabular}{|c|c|c|c|c|c|c|}
\hline Título & Autores & Ano & Periódico & Abordagem & Método & Principais resultados \\
\hline $\begin{array}{c}\text { An integrated model } \\
\text { to assess the } \\
\text { leanness and agility } \\
\text { of the automotive } \\
\text { industry }\end{array}$ & $\begin{array}{c}\text { Azevedo et } \\
\text { al. }\end{array}$ & 2012 & $\begin{array}{l}\text { Resources, } \\
\text { Conservation, } \\
\text { and Recycling }\end{array}$ & Quantitativa & $\begin{array}{l}\text { Estudo de } \\
\text { Caso }\end{array}$ & $\begin{array}{l}\text { Os autores utilizam o índice } \\
\text { Agilean em uma cadeia de } \\
\text { fornecimento. Este índice } \\
\text { permite avaliar as empresas } \\
\text { e suas respectivas cadeias } \\
\text { de suprimentos, sendo } \\
\text { traduzidas em pontuações } \\
\text { para comparações futuras } \\
\text { com empresas concorrentes. }\end{array}$ \\
\hline $\begin{array}{c}\text { An integrated } \\
\text { stochastic-fuzzy } \\
\text { modeling approach } \\
\text { for supply chain } \\
\text { leanness evaluation }\end{array}$ & $\begin{array}{l}\text { Behrouzi e } \\
\text { Wong }\end{array}$ & 2013 & $\begin{array}{l}\text { International } \\
\text { Journal of } \\
\text { Advanced } \\
\text { Manufacturing } \\
\text { Technology }\end{array}$ & Quantitativa & $\begin{array}{l}\text { Estudo de } \\
\text { Caso }\end{array}$ & $\begin{array}{l}\text { Utilizando o modelo da } \\
\text { lógica fuzzy em indústrias } \\
\text { automotivas, o método tem } \\
\text { como foco quantificar o } \\
\text { grau de enxugamento em } \\
\text { quatro categorias de } \\
\text { desempenho: qualidade, } \\
\text { custo, entrega e } \\
\text { confiabilidade e } \\
\text { flexibilidade. }\end{array}$ \\
\hline $\begin{array}{c}\text { Application of } \\
\text { artificial neural } \\
\text { network for fuzzy } \\
\text { logic based leanness } \\
\text { assessment }\end{array}$ & $\begin{array}{l}\text { Vimal e } \\
\text { Vinodh }\end{array}$ & 2013 & $\begin{array}{c}\text { Journal of } \\
\text { Manufacturing } \\
\text { Technology } \\
\text { Management }\end{array}$ & Quantitativa & $\begin{array}{l}\text { Estudo de } \\
\text { Caso }\end{array}$ & $\begin{array}{c}\text { O estudo utiliza a } \\
\text { ferramenta ANN, para } \\
\text { melhorar a eficácia da } \\
\text { computação, na aplicação } \\
\text { do modelo da lógica fuzzy. } \\
\text { A ferramenta foi modelada } \\
\text { e simulada, utilizando o } \\
\text { software MATLAB. }\end{array}$ \\
\hline $\begin{array}{l}\text { Leanness estimation } \\
\text { procedural } \\
\text { hierarchy using } \\
\text { interval-valued fuzzy } \\
\text { sets (IVFS) }\end{array}$ & $\begin{array}{l}\text { Matawale, } \\
\text { Datta e } \\
\text { Mahapatra }\end{array}$ & 2014 & $\begin{array}{c}\text { Benchmarking: } \\
\text { An International } \\
\text { Journal }\end{array}$ & $\begin{array}{l}\text { Qualitativa e } \\
\text { Quantitativa }\end{array}$ & $\begin{array}{l}\text { Estudo de } \\
\text { Caso }\end{array}$ & $\begin{array}{l}\text { Avaliou o desempenho da } \\
\text { produção enxuta em uma } \\
\text { indústria na Índia, bem } \\
\text { como identificou as áreas } \\
\text { com os piores índices. O } \\
\text { estudo apresenta o sistema } \\
\text { de lógica fuzzy baseado em } \\
\text { números fuzzy trapezoidais. }\end{array}$ \\
\hline
\end{tabular}

Fonte: Elaborado pelos autores.

A partir dos artigos selecionados, foi possível observar a evolução da quantidade de publicações durante o passar dos anos (Figura 2). Embora o ano de 1997 tenha sido estabelecido como data inicial do período de busca, o primeiro artigo encontrado datava de 2002. Com isso, pode-se concluir que o tema é recente e possui uma pequena quantidade de publicações que apresenta uma tendência crescente, indicando que o desenvolvimento de modelos de avaliação da implantação da produção enxuta ainda pode ser bastante explorado.

No entanto, com a pequena quantidade de publicações relacionadas ao tema, pode-se constatar que a avaliação da produção enxuta vem sendo, de certa forma negligenciada pelos pesquisadores. Esse fato pode dificultar a implementação da produção enxuta, uma vez que o processo é longo e necessita de instrumentos para acompanhar sua evolução. 
Com relação aos periódicos nos quais os artigos selecionados foram publicados, pode-se verificar que a maior quantidade de publicações ocorreu nos periódicos “International Journal of Advanced Manufacturing Technology", "Journal of Manufacturing Technology Management" e "International Journal of Production Research" com cinco, três e duas publicações respectivamente, como pode ser observado na Tabela 3.

Figura 2-Quantidade de artigos publicados por ano.

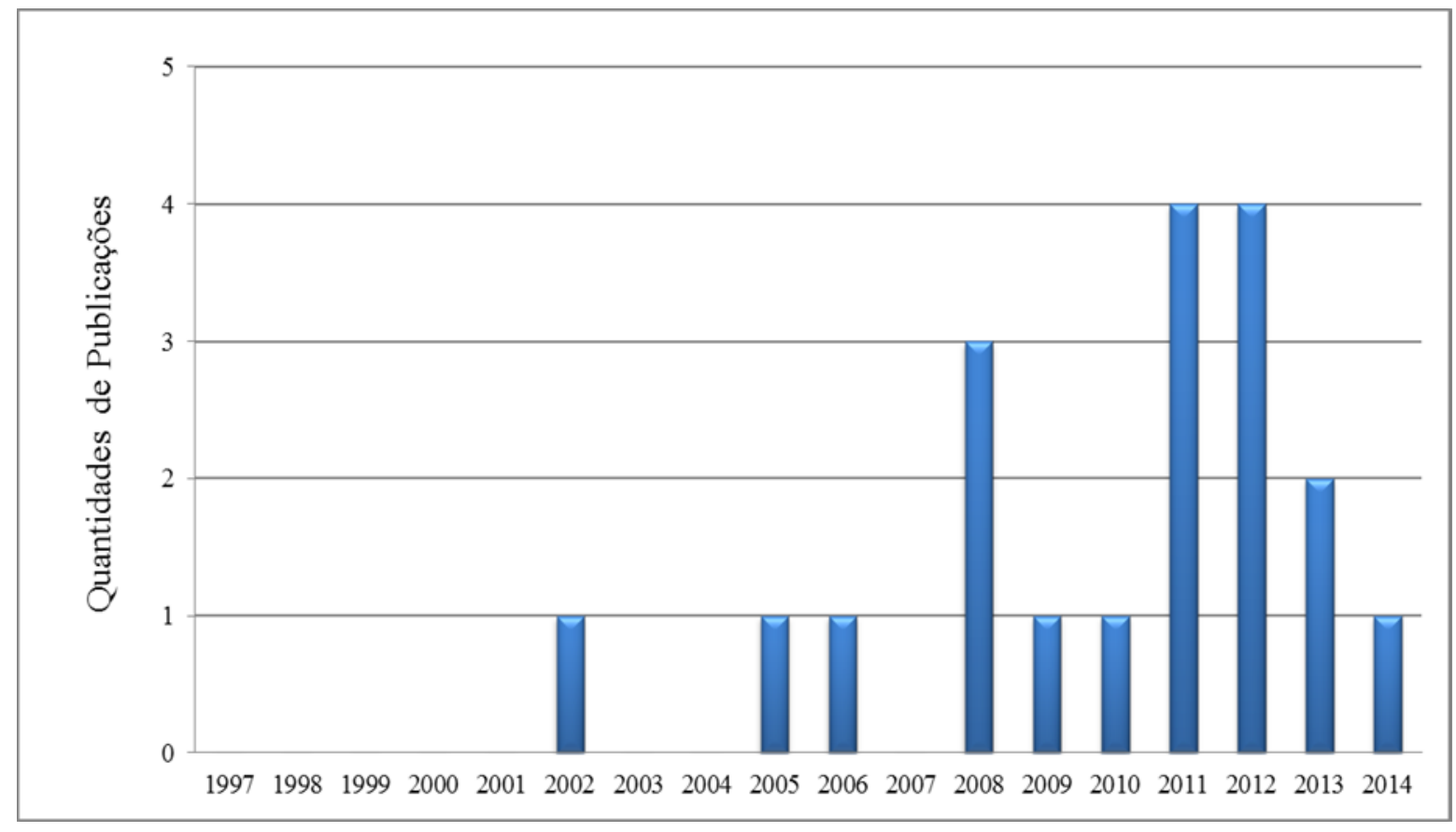

Fonte: Elaborada pelos autores.

Tabela 3 - Quantidade de artigos por periódico

\begin{tabular}{lc}
\hline Periódico & Quantidade de artigos \\
\hline International Journal of Advanced Manufacturing Technology & 5 \\
Journal of Manufacturing Technology Management & 3 \\
International Journal of Production Research & 2 \\
Integrated Manufacturing Systems & 1 \\
Management Decision & 1 \\
Journal of Engineering and Technology Management & 1 \\
Computers in Industry & 1 \\
Measuring Business Excellence & 1 \\
International Journal of Lean Six Sigma & 1 \\
Expert Systems with Applications & 1 \\
Benchmarking: An International Journal & 1 \\
Resources, Conservation, and Recycling & 1 \\
\hline TOTAL & 19 \\
\hline
\end{tabular}

Fonte: Elaborada pelos autores. 
Com relação à abordagem de pesquisa adotada pelos artigos selecionados, pode-se observar uma predominância pela abordagem quantitativa no desenvolvimento dos métodos de avaliação. Essa constatação pode ser corroborada pela utilização da lógica fuzzy na maioria dos casos, a qual necessita de procedimentos matemáticos para ser operacionalizada. Além da lógica fuzzy, outras técnicas quantitativas, como a análise envoltória de dados (DEA), também foram utilizadas.

Quanto ao procedimento de pesquisa utilizado, verificou-se que o estudo de caso foi o escolhido pela maior parte das publicações, totalizando treze dentre os dezenove artigos. O restante se encontra distribuído entre survey e modelagem, apresentando quatro e duas ocorrências respectivamente.

O procedimento do estudo de caso foi amplamente utilizado nas pesquisas, inclusive nas que possuíam o caráter quantitativo em seu método de pesquisa. Uma vez que os métodos de avaliação geralmente são quantitativos, o uso do estudo de casos pode se justificar pela necessidade de testar o método desenvolvido em contextos reais.

\section{Contribuição dos métodos de avaliação da produção enxuta}

Após a classificação dos artigos que proporcionou uma visão panorâmica do portfólio selecionado, foi realizada uma revisão do conteúdo dos trabalhos publicados, a fim de verificar a contribuição de cada um deles para a literatura da área. A seguir são apresentadas as ideias principais dos trabalhos selecionados de acordo com a ordem cronológica de publicação.

O modelo proposto por Soriano-Meier e Forrester (2002) busca avaliar a adoção dos princípios da produção enxuta utilizando variáveis semelhantes às do modelo desenvolvido por Karlsson e Åhlström (1996), são elas: eliminação de desperdícios, melhoria contínua, zero defeitos, entregas just in time, produção puxada, equipes multifuncionais, descentralização, integração de funções e sistemas de informações verticais. Além disso, o modelo também visa medir o compromisso da gerência com a produção enxuta com base em um modelo desenvolvido por Boyer (1996). O principal instrumento de pesquisa utilizado foi um survey, fazendo uso também de entrevistas estruturadas, dados secundários internos e externos e observações in loco. $\mathrm{O}$ questionário desenvolvido para avaliar o grau de adoção aos princípios da produção enxuta foi elaborado com base nas nove variáveis identificadas por Karlsson e Åhlström (1996) e o questionário desenvolvido para avaliar o compromisso da gestão com a produção enxuta utilizou o modelo de Boyer (1996) com pequenas modificações. Ambos solicitavam que os entrevistados classificassem as respostas em uma escala de Likert de sete pontos. Embora relevante para a pesquisa científica, o modelo de Soriano-Meier e Forrester (2002) não foi desenvolvido para fins de aplicação prática. Portanto, não parece viável a sua utilização como instrumento de autoavaliação. 
O método utilizado por Taj $(2005,2008)$ busca avaliar nove principais áreas da produção, são elas: estoque; trabalho em equipe; processos; manutenção; layout e movimentação; fornecedores; setup; qualidade; e planejamento e controle. A ferramenta foi aplicada em fábricas na China por meio de um questionário, no qual os entrevistados foram convidados a responder de 3 a 6 perguntas sobre cada área, sendo fornecida uma pontuação de 0 a 4 para cada resposta. Posteriormente, as pontuações foram totalizadas para cada área e um gráfico de perfil enxuto foi criado para ilustrar resultados. Na verdade, o método utilizado por Taj $(2005,2008)$ foi desenvolvido por Lee (2015), com o propósito de fornecer um diagnóstico rápido do estágio de desenvolvimento da produção enxuta. Esse método, no entanto, é mais focado em indicadores de desempenho (resultados) do que em práticas enxutas (ações), o que pode dar uma noção imprecisa do grau de enxugamento (leanness).

Srinivasaraghavan e Allada (2006) desenvolveram um instrumento de pesquisa para coletar informações necessárias para a avaliação do grau de implementação da produção enxuta na empresa, buscando fornecer uma medida quantitativa por meio da comparação de outras indústrias enxutas. O método utiliza a distância de Mahalanobis que é uma medida de distância baseada em correlações entre variáveis, na qual é possível identificar e analisar diferentes padrões tomando uma referência como base. A proposta consiste em quatro etapas: coleta de dados utilizando ferramentas contemporâneas de avaliação; normalização dos dados; cálculo da distância de Mahalanobis; identificação e direção das melhorias de acordo com o orçamento da empresa. A desvantagem deste método é que a avaliação é sempre comparativa, o que demanda a necessidade de escolher empresas de referência para a aplicação.

Bayou e Korvin (2008), por meio de estudos de caso, apresentam os passos de um algoritmo utilizando a lógica fuzzy e aplica-o à indústria automobilística. Os autores buscam uma medida unificadora dos efeitos das práticas da produção enxuta, uma medida que integre as mudanças ocorridas decorrentes das práticas em um indicador, fazendo com que duas empresas concorrentes possam ser comparadas a fim de determinar o grau de enxugamento de suas operações. Para avaliar o grau de enxugamento das operações os autores utilizam um conjunto de práticas que julgam ser relevantes para uma empresa tornar-se enxuta, são elas: JIT, Kaizen e Gestão da Qualidade. Ao final, a empresa é classificada como enxuta, medianamente enxuta ou muito enxuta. Assim como no método de Srinivasaraghavan e Allada (2006), o método de Bayou e Korvin (2008) depende de uma empresa que possa servir de benchmark para a avaliação.

Wan e Chen (2008) propõem um método para medir o quão enxuto se encontram os sistemas de manufatura por meio da Análise Envoltória de Dados (DEA). Com isso, fornecem uma maneira de quantificar o grau de enxugamento de um sistema de produção baseado em uma referência ideal obtida a partir de dados históricos. Os sistemas de produção atuais são denominados 
de Unidades Tomadoras de Decisões Atuais (UTDA) e os sistemas enxutos viáveis são chamados de Unidades Tomadoras de Decisões Ideais (UTDI). As pontuações demonstram o quão enxuto é o sistema e cria diferentes cenários que avaliam o trade-off entre três dimensões (custo, lead time produtivo e valor agregado ao produto) a fim de proporcionar informações de apoio à tomada de decisão alinhada com o foco estratégico da empresa.

Wan e Chen (2009) também propuseram uma ferramenta de apoio à decisão adaptável aos sistemas produtivos das empresas que foi desenvolvido na busca de orientar as mesmas no processo de implementação da produção enxuta. A partir da ferramenta, desenvolvem um software que auxilia na avaliação do estado atual da empresa, na identificação dos pontos de melhoria e gera sugestões sobre as ferramentas e técnicas que devem ser utilizadas para os planos de ação das melhorias. Para que a produção enxuta seja implementada de forma sistemática e eficiente a ferramenta faz a associação do conhecimento e da experiência de seus profissionais com ações práticas buscando responder a dois questionamentos: "Quão enxuto o sistema é?" e "Como se tornar mais enxuto?".

Singh, Garg e Sharma (2010) apresentam uma discussão sobre os conceitos lean e elaboram um método para medir o quanto uma empresa é considerada enxuta. Para isso, foi selecionada uma equipe com cinco membros com experiência em implementação da produção enxuta. Cada membro da equipe foi solicitado a classificar a situação atual da empresa de acordo com cinco parâmetros, a saber: fornecedor, prioridades de investimento, práticas da produção enxuta, desperdícios e problemas com clientes. A equipe determinou uma escala de medida variando de aceitável a totalmente satisfatória. Posteriormente, a lógica fuzzy é introduzida na busca de remover o viés do julgamento humano. Além disso, foram atribuídas pontuações diferentes para os avaliadores. Ao final, a partir dos dados obtidos, é gerado o grau enxugamento da organização.

Bhasin (2011) avalia a produção enxuta por meio de um modelo composto por 104 índices agrupados em 12 categorias. Com o objetivo de avaliar a viabilidade do modelo, o mesmo foi aplicado em 20 organizações avaliando as 12 categorias com o respectivo conjunto de índices para cada grupo. A partir das pontuações obtidas na avaliação as organizações são classificadas em sete fases, a saber: planejamento, desenvolvimento, mecânica, aprimorada, holística, inovadora e ideológica. Embora fácil de aplicar, o método de Bhasin (2011) é pouco orientado para a prática e não deixa claro o caminho de melhoria a ser seguido a partir do diagnóstico.

No artigo de Seyedhosseini et al. (2011), os autores buscam medir o desempenho da produção enxuta usando a estrutura do Balanced Scorecard. Para isso, os autores identificaram cinco perspectivas: perspectiva financeira, perspectiva do cliente, perspectiva dos processos, perspectiva das pessoas e perspectiva dos fornecedores. Posteriormente, são gerados 16 objetivos enxutos com relação a cada perspectiva usando a opinião de especialistas por meio do método 
Delphi. Em seguida, foram determinados critérios adequados e medidas de desempenho usando o Balanced Scorecard, gerando 52 critérios. Foi utilizada a técnica Decision Making Trial and Evaluation Laboratory (DEMATEL) juntamente com a opinião dos especialistas para elaborar uma matriz representando a importância e a prioridade entre os objetivos e as medidas enxutas. Com isso foi possível identificar uma relação de causa e efeito entre os objetivos e desenhar um mapa de estratégia enxuta para a organização.

No estudo realizado por Vinodh, Prakash e Selvan (2011) foi utilizada a lógica fuzzy associada à técnica de mineração de dados, a FARM (Fuzzy Association Rules Mining). FARM é uma técnica de mineração de dados que busca extrair apenas as informações úteis, essa técnica foi aplicada aos bancos de dados das empresas pesquisadas. $\mathrm{O}$ método utiliza atributos para avaliar a produção enxuta que foram coletados baseados em unidades de decisão quantitativa (custo) e qualitativa (rentabilidade, produtividade, qualidade, lead time, defeitos e disponibilidade). Esses atributos são as medidas de desempenho da produção enxuta e foram aprovados pelos tomadores de decisão da organização que afirmaram que esses atributos são relevantes para a avaliação das práticas da produção enxuta.

Vinodh e Chintha (2011), Vinodh e Vimal (2012) e Vinodh e Kumar (2012) apresentam um modelo conceitual composto por cinco facilitadores enxutos, 20 critérios enxutos associados aos facilitadores e vários atributos que são atrelados aos critérios. Os facilitadores foram classificados da seguinte maneira: responsabilidade da gestão, administração da produção, força de trabalho, tecnologia e estratégia de manufatura.

No artigo de Vinodh e Chintha (2011) foram escolhidos cinco especialistas em produção enxuta para a avaliação, os especialistas foram orientados a escolher uma entre cinco notas para cada critério, sendo elas: 10, 8, 6, 4 e 2. De 8 a 10 corresponde a extremamente enxuta, de 6 a 8 é enxuta, de 4 a 6 é classificada como geralmente enxuta, de 2 a 4 é não enxuta e menos do que 2 representa extremamente não enxuta. Posteriormente, é aplicada a lógica fuzzy e a partir das notas é gerado o grau de enxugamento da empresa.

Já no artigo de Vinodh e Vimal (2012), foi solicitado que os chefes de vários departamentos da organização respondessem questões para avaliar o desempenho das capacidades enxutas e a importância dos pesos das capacidades enxutas usando variáveis linguísticas.

No modelo de Vinodh e Kumar (2012) é desenvolvido um sistema de apoio à decisão (DSS) para a avaliação do grau de enxugamento pela lógica fuzzy, o DSS-MGFLA. O DSS avalia vários graus de enxugamento e permite que os gestores tomem decisões apropriadas do ponto de vista da avaliação do grau de enxugamento e melhorias. Foram desenvolvidos sistemas para controle em tempo real de processos de fabricação, para a gestão da cadeia de suprimentos, gestão de 
relacionamentos com os clientes, tomada de decisões em gestão de projetos, segurança de transporte e decisões sobre fazer ou comprar.

Tanto o modelo desenvolvido por Vinodh e Chintha (2011), como os de Vinodh e Vimal (2012) e Vinodh e Kumar (2012) utilizam a lógica fuzzy para a aproximação das variáveis linguísticas e para calcular o índice do grau de enxugamento.

O objetivo principal do trabalho de Vimal e Vinodh (2012) foi avaliar o grau de enxugamento usando regras da abordagem IF-THEN juntamente com relevância prática. Inicialmente foi realizada uma avaliação do desempenho dos recursos da produção enxuta selecionando as variáveis linguísticas, para posteriormente desenvolver um conjunto de números fuzzy para aproximar os valores das variáveis linguísticas. Após a obtenção dos números fuzzy, regras IF-THEN são aplicadas para o cálculo do índice de enxugamento. Como resultado, o artigo apresenta o desenvolvimento de um modelo abrangente de avaliação do grau de enxugamento, usando a lógica fuzzy baseada no mecanismo IF-THEN.

Azevedo et al. (2012) desenvolveram um método para calcular um índice que denominaram de Agilean. O método permite avaliar a agilidade e o grau de enxugamento das empresas e suas respectivas cadeias de suprimentos, sendo traduzidas em pontuações para comparações futuras com empresas concorrentes. O método inclui a opinião de especialistas por meio do método Delphi e foi testado empiricamente para conferir se a aplicação do índice retoma com valores significativos, agilidade e grau de enxugamento, capazes de modificar e aprimorar a tomada de decisão.

Behrouzi e Wong (2013) propõem avaliar o grau de enxugamento, por meio da lógica fuzzy, em pequenas e médias empresas na indústria automotiva. Quatro categorias de desempenho (qualidade, custo, entrega e confiabilidade e flexibilidade) foram selecionadas, juntamente com 28 medidas relacionadas para avaliar o grau de enxugamento e, posteriormente, sugerir algumas ações de gerenciamento com base nos diferentes valores de níveis de enxugamento.

No artigo de Vimal e Vinodh (2013) foi utilizada a ANN (Artificial Neural Network) integrada à lógica fuzzy para a determinação do grau de enxugamento em uma empresa na Índia. Com objetivo de melhorar a eficácia da computação (manual), a rede foi modelada e simulada, utilizando o software MATLAB. Um estudo de caso foi realizado para validar o modelo.

O artigo de Matawale, Datta e Mahapatra (2014) desenvolve um método para avaliação do desempenho de enxugamento em uma indústria na Índia, com objetivo de identificar as áreas com os piores índices. Foi utilizado um sistema de lógica fuzzy baseado em números fuzzy trapezoidais (IV), que resultou num sistema de apoio à decisão, indo além da avaliação do grau de implementação da produção enxuta.

Diante do que foi pesquisado e analisado percebe-se que as publicações analisadas apontam de uma forma geral para a avaliação da produção enxuta utilizando ferramentas quantitativas como 
a lógica fuzzy (predominantemente) e a análise envoltória de dados (DEA). A grande maioria dos trabalhos desenvolve um modelo conceitual e aplica-o em uma organização como meio de validação. Na maioria das vezes, sugerem áreas nas quais a implementação pode ser melhorada.

Ao analisar os artigos, fica evidente que os métodos para avaliação da produção enxuta continuam em evolução. Em uma leitura detalhada dos artigos mais recentes é possível observar que eles constroem seus métodos a partir de métodos mais antigos, confirmando o processo natural de evolução da pesquisa científica. Contudo, mesmo reconhecendo o valor das contribuições já realizadas, é possível identificar lacunas na literatura, uma vez que a pesquisa nessa temática ainda pode ser considerada recente.

\section{Identificação de lacunas e perspectivas futuras}

Com base na leitura e na análise dos artigos selecionados pode-se identificar pontos relevantes para o aprofundamento do tema, bem como lacunas que representam oportunidades de pesquisas futuras para o desenvolvimento de novos métodos de avaliação da implementação da produção enxuta em empresas.

Ao pesquisar a literatura, os autores procuraram identificar parâmetros para avaliar os artigos selecionados. Esses parâmetros foram definidos como requisitos necessários para um método de avaliação do grau de implementação da produção enxuta. De acordo com o levantamento realizado, foram identificados os seguintes requisitos:

- Adequação ao sistema produtivo. O questionamento sobre a universalidade da produção enxuta (JAMES-MOORE; GIBBONS, 1997; COONEY, 2002) fez surgir uma corrente de pesquisas que estudam as adaptações da produção enxuta para diferentes tipos de sistemas produtivos (LANDER; LIKER, 2007; LEE; JO, 2007; DEFLORIN; SCHERRER-RATHJE, 2012). Isso significa que cada sistema de produção pode ter objetivos e necessidades diferentes ao implementar a produção enxuta, o que reflete diretamente na forma pela qual o sistema é avaliado. O grau de implementação das práticas de produção enxuta pode ser diferente entre as empresas devido às diferenças entre os sistemas de produção. Em outras palavras, a implementação completa de uma determinada prática pode ser necessária em uma empresa e desnecessária em outra. Portanto, o método de avaliação deve ser adaptativo, de maneira que seja possível adequá-lo às características do sistema produtivo a ser avaliado.

- Avaliação ponderada das práticas. A produção enxuta é operacionalizada por um conjunto de práticas específicas de gestão da produção que trabalham de maneira sinérgica, complementando umas às outras. Portanto, a interação entre as práticas enxutas deve ser considerada como essencial para o sucesso da produção enxuta nas empresas. Dessa forma, torna-se essencial a 
avaliação do grau de implementação das práticas de produção enxuta a partir de uma avaliação ponderada entre as práticas e sobre sua relação com as características do sistema produtivo. $\mathrm{O}$ método deve considerar que os benefícios decorrentes de determinada prática enxuta dependem da interação da mesma com o sistema produtivo e com as outras práticas, sendo que a aplicação isolada não garante a implementação por completo. Para isso as práticas devem ser avaliadas por meio de forma ponderada, considerando as inter-relações e as características do sistema produtivo da empresa.

- Níveis de implementação. A produção enxuta deve ser vista como uma direção a ser seguida, ou seja, é um objetivo em andamento e não um estado a ser alcançado. Portanto, para ser capaz de estudar esse processo de mudança é preciso medir as melhorias alcançadas e identificar o estágio de maturidade que a empresa se encontra. Deste modo, além de implementar a produção enxuta, torna-se imprescindível a avaliação do seu progresso e os ganhos alcançados (KARLSSON; ÅHLSTRÖM, 1996). Diante disso, torna-se essencial o estabelecimento de níveis de implementação da produção enxuta. O método deve estabelecer níveis que demonstrem o desempenho da empresa, revelando o estado atual rumo à produção enxuta.

- Usabilidade. O método deve ser fácil de usar e possuir uma boa interação com os gerentes para que os mesmos possam utilizar e compreender o método rapidamente. A usabilidade do método faz com que o mesmo possa sair do plano acadêmico e passe a ser aplicado no âmbito das próprias empresas, como instrumento de autoavaliação.

Tomando como parâmetros os requisitos necessários para um método de avaliação do grau de implementação da produção enxuta, o Quadro 2 confronta os métodos analisados com os requisitos propostos.

Analisando o Quadro 2, observa-se que os métodos revisados não atendem completamente aos requisitos listados. Em confronto com os requisitos, o método proposto por Wan e Chen (2009) mostrou-se superior aos demais. Esse método atende completamente ao requisito "nível de implementação" e parcialmente aos requisitos "adequação ao sistema produtivo" e "usabilidade". Com relação à adequação ao sistema produtivo o método de Wan e Chen (2009) considerou o volume de produção e a repetitividade do processo, porém negligenciou a influência que as oscilações da demanda podem desempenhar na operacionalização da produção enxuta. Com relação à usabilidade pode-se afirmar que a interface do software que foi desenvolvido torna o método de fácil manuseio, no entanto, ele ainda apresenta dificuldades para os usuários realizarem modificações e replicarem o estudo em seus ambientes produtivos.

O não atendimento aos requisitos propostos aqui evidencia as lacunas que ainda não foram preenchidas pelos métodos atuais e sinaliza um caminho para pesquisas futuras. A necessidade de adequação ao sistema produtivo é a primeira linha de pesquisa que surge a partir da análise 
realizada. À medida que as empresas evoluem na implementação da produção enxuta, elas podem desenvolver sistemas de gestão da produção específicos (NETLAND, 2013), mais adequados ao seu contexto e às circunstâncias particulares que enfrentam. Com isso, os métodos de avaliação que consideram um sistema enxuto "puro" como referência perdem o sentido nesse caso, pois a referência pode ter mudado. Daí surge a necessidade de se dispor de avaliações adaptativas, tal como se propõem Wan e Chen (2009).

Quadro 2 - Comparação dos modelos com os requisitos propostos.

\begin{tabular}{|c|c|c|c|c|c|}
\hline & & \multicolumn{4}{|c|}{ Requisitos } \\
\hline & & 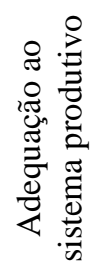 & 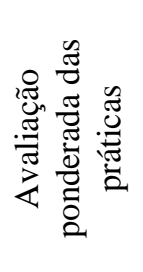 & 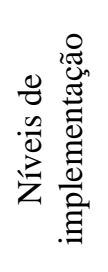 & 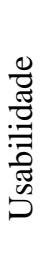 \\
\hline \multirow{18}{*}{ 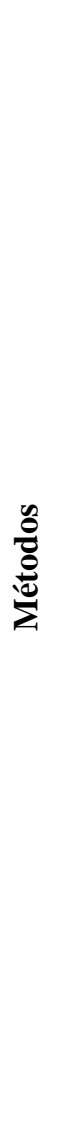 } & Soriano-Meier e Forrester (2002) & & & & \\
\hline & Taj $(2005,2008)$ & & & & \\
\hline & Srinivasaraghavan e Allada (2006) & & & & \\
\hline & Bayou e Korvin (2008) & & & & \\
\hline & Wan e Chen (2008) & & & & \\
\hline & Wan e Chen (2009) & & & & \\
\hline & Singh, Garg e Sharma (2010) & & & & \\
\hline & Bhasin (2011) & & & & \\
\hline & Seyedhosseini et al. (2011) & & & & \\
\hline & Vinodh, Prakash e Selvan (2011) & & & & \\
\hline & Vinodh e Chintha (2011) & & & & \\
\hline & Vinodh e Vimal (2012) & & & & \\
\hline & Vinodh e Kumar (2012) & & & & \\
\hline & Vimal e Vinodh (2012) & & & & \\
\hline & Azevedo et al. (2012) & & & & \\
\hline & Behrouzi e Wong (2013) & & & & \\
\hline & Vimal e Vinodh (2013) & & & & \\
\hline & Matawale, Datta e Mahapatra (2014) & & & & \\
\hline & \multicolumn{4}{|l|}{ Atende completamente ao requisito } & \\
\hline & \multicolumn{4}{|l|}{ Atende parcialmente ao requisito } & \\
\hline & Não atende ao requisito & & & & \\
\hline
\end{tabular}

Fonte: Elaborado pelos autores.

A ausência do atendimento ao requisito de avaliação ponderada das práticas mostra que o caráter sistêmico da produção enxuta, muito discutido na literatura, não foi refletido para os 
métodos de avaliação. Explorar a interação entre as práticas e suas inter-relações com as características do sistema de produção é uma funcionalidade que merece ser pesquisada no desenvolvimento de novos métodos de avaliação.

O requisito de classificar o sistema avaliado em níveis de implementação tem sido razoavelmente atendido pelos métodos atuais. No entanto, ainda é necessário que as pesquisas futuras abordem melhor a relação entre o nível em que a empresa se classifica e as ações de melhoria que devem ser desempenhadas para que a mesma continue evoluindo. Um dos grandes desafios da produção enxuta é a manutenção do sistema após a implementação. Estabelecer a ligação entre o subsistema de avaliação e os planos de ação pode fomentar a cultura de melhoria contínua para a manutenção do sistema.

Por maior que seja a sofisticação de um método, não se pode ignorar que a pesquisa nesta área é aplicada e seus resultados não podem ignorar a contribuição prática que se espera. Portanto, o método de avaliação deve mostrar viabilidade de uso, à medida que os seus usuários podem aplicálo livremente sem a necessidade de um treinamento mais complexo ou da contratação de profissionais especializados externos à empresa. Assim, um dos grandes desafios para futuros métodos é equilibrar a precisão na avaliação com a usabilidade, permitindo a fácil disseminação dos instrumentos propostos.

\section{Considerações finais}

Este artigo procurou analisar os métodos atuais para a avaliação do grau de implementação da produção enxuta. Para isso, reuniu um conjunto de 19 artigos publicados entre 2002 e 2014, que foram selecionados por uma busca sistemática na literatura pertinente à área. Os artigos foram analisados, suas contribuições foram identificadas e as lacunas deixadas na literatura puderam ser observadas pela análise agregada do portfólio selecionado. As lacunas identificadas sugeriram perspectivas futuras de pesquisa, considerando que o tema ainda está em desenvolvimento.

Por um lado, constatou-se que, embora seja um tema relativamente recente, os métodos atuais apresentam um grau de sofisticação que possibilita avaliações detalhadas do estágio de maturidade da produção enxuta nas empresas. Por outro lado, o grau de sofisticação pode comprometer a usabilidade do método, já que sua finalidade sempre está associada à possibilidade de aplicação prática.

Outro ponto que merece destaque é que os métodos atuais parecem não acompanhar a tendência de adaptação da produção enxuta às características contingenciais de cada sistema produtivo. Avaliar diferentes sistemas produtivos como se a necessidade da implementação práticas 
de produção enxuta fosse igual e invariável pode constituir um erro que leva a diagnósticos que não conduzem à melhoria real da organização.

A dificuldade dos métodos atuais em abordar a interação entre as práticas de produção enxuta e a relação dessas práticas com as características de cada sistema produtivo também foi observada nesta pesquisa. Abordar essa limitação constitui um desafio, mas também um campo frutífero para pesquisas futuras.

Com a revisão bibliográfica realizada foi possível adquirir um conhecimento mais abrangente sobre o tema, bem como compreender os métodos que já foram propostos, a fim de aproveitar suas contribuições para a elaboração de novos métodos de avaliação. Assim, conclui-se que este artigo cumpriu sua finalidade, pois, ao identificar elementos pouco abordados nos métodos atuais, abriu um vasto campo a ser explorado que direciona o futuro dos métodos de avaliação da produção enxuta.

\begin{abstract}
One of the critical success factors for the maintenance of lean production is the use of methods to assess the evolution of the system. However, the scientific literature on the assessment methods is still at an early stage, lacking a systematization of the literature in order to rise the state of the art on the subject. Given this need, this paper aims to review the literature on methods for lean production assessment in order to systematize the knowledge already published and identify research gaps that may guide the future development of the subject. For this, systematic review procedures were adopted for the search and analysis of articles, restricting the scope for identifying useful features for developing methods for lean production assessment. The data gathering generated a portfolio of 19 articles published between 2002 and 2014. Selected papers presented assessment methods that were analyzed according to four parameters: suitability to the productive system, weighted assessment of practices, identification of implementation levels and usability. The results show that current methods have sophisticated procedures and continue to evolve. On the other hand, it can observed that the adaptation to the productive system and the systemic and weighted assessment of practices are requirements still neglected and that indicate the paths for future research.
\end{abstract}

Key-words: lean production; assessment; literature review.

\title{
Referências
}

AZEVEDO, S. G. et al. An integrated model to assess the leanness and agility of the automotive industry. Resources, Conservation and Recycling, v. 66, n. 1, p. 85-94, 2012. crossref

BAMFORD, D. et al. Partial and iterative lean implementation: two case studies. International Journal of Operations \& Production Management, v. 35, n. 5, p. 702-727, 2015. crossref

BAYOU, M. E.; KORVIN, A. Measuring the leanness of manufacturing systems: a case study of Ford Motor Company and General Motors. Journal of Engineering and Technology Management, v. 25, n. 4, p. 287-304, 2008. cross ref

BEHROUZI, F.; WONG, K. Y. An integrated stochastic-fuzzy modeling approach for supply chain leanness evaluation. International Journal of Advanced Manufacturing Technology, v. 68, n. 5-8, p. 1677-1696, 2013. crossref

BHAMU, J.; SANGWAN, K. S. Lean manufacturing: literature review and research issues. International Journal of Operations \& Production Management, v. 34, n. 7, p. 876-940, 2014. crossref 
BHASIN, S. Measuring the leanness of an organisation. International Journal of Lean Six Sigma, v. 2, n. 1, p. 55-74, 2011. cross ref

BOYER, K. K. Longitudinal linkages between intended and realized operations strategies. International Journal of Operations \& Production Management, v. 18, n. 4, p. 356-373, 1996. crossref

CAMACHO-MIÑANO, M.; MOYANO-FUENTES, J.; SACRISTÁN-DÍAZ, M. What can we learn from the evolution of research on lean management assessment? International Journal of Production Research, v. 51, n. 4, p. 10981116, 2013. crossref

COONEY, R. Is "lean" a universal production system? Batch production in the automotive industry. International Journal of Operations \& Production Management, v. 22, n. 10, p. 1130-1147, 2002. cross ref

CORDEIRO, A. M. et al. Revisão sistemática: uma revisão narrativa. Revista do Colégio Brasileiro de Cirurgiões, v. 34 , n. 6, p. 428-431, 2007. crossref

DEFLORIN, P.; SCHERRER-RATHJE, M. Challenges in the transformation to lean production from different manufacturing-process choices: a path-dependent perspective. International Journal of Production Research, v. 50, n. 14 , p. 3956-3973, 2012. cross ref

DE-LA-TORRE-UGARTE-GUANILO, M. C.; TAKAHASHI, R. F.; BERTOLOZZI, M. R. Revisão sistemática: noções gerais. Revista da Escola de Enfermagem da USP, v. 45, n. 5, p. 1260-1266, 2011. crossref

JAMES-MOORE, S. M.; GIBBONS, A. Is lean manufacture universally relevant? An investigative methodology. International Journal of Operations \& Production Management, v. 17, n. 9, p. 899-911, 1997. crossref

JASTI, N. V. K.; KODALI, R. Lean production: literature review and trends. International Journal of Production Research, v. 53, n. 3, p. 867-885, 2015. crossref

KARLSSON, C.; ÅHLSTRÖM, P. Assessing changes towards lean production. International Journal of Operations \& Production Management, v. 16, n. 2, p. 24-41, 1996. crossref

LANDER, E.; LIKER, J. K. The Toyota Production System and art: making highly customized and creative products the Toyota way. International Journal of Production Research, v. 45, n. 16, p. 3681-3698, 2007. crossref

LEE, B-H.; JO, H-J. The mutation of the Toyota Production System: adapting the TPS at Hyundai Motor Company. International Journal of Production Research, v. 45, n. 16, p. 3665-3679, 2007.

LEE, Q. Assessment for lean manufacturing: a benchmarking tool. Disponível em: <http://www.strategosinc.com/assessment.htm>. Acesso em: 04 out. 2015.

MARODIN, G.; SAURIN, T. A. Implementing lean production systems: research areas and opportunities for future studies. International Journal of Production Research, v. 51, n. 22, p. 6663-6680, 2013. crossref

MARTINS, R. A. Abordagens quantitativa e qualitativa. In: MIGUEL, P. A. C. (org.). Metodologia de pesquisa em Engenharia de Produção e Gestão de Operações. Rio de Janeiro: Elsevier, p. 46-61, 2010.

MATAWALE, C. R; DATTA, S.; MAHAPATRA, S. S. Leanness estimation procedural hierarchy using intervalvalued fuzzy sets (IVFS). Benchmarking: An International Journal, v. 21, n. 2, p. 150-183, 2014.

NETLAND, T. Exploring the phenomenon of company-specific production systems: one-best-way or own-best-way? International Journal of Production Research, v. 51, n. 4, p. 1084-1097, 2013. crossref

NETLAND, T. H.; SCHLOETZER, J. D.; FERDOWS, K. Implementing corporate lean programs: the effect of management control practices. Journal of Operations Management, v. 36, p. 90-102, 2015. cross ref

SEYEDHOSSEINI, S. M. et al. Extracting leanness criteria by employing the concept of Balanced Scorecard. Expert Systems with Applications, v. 38, p. 10454-10461, 2011. crossref

SINGH, B.; GARG, S. K.; SHARMA, S. K. Development of index for measuring leanness: study of an Indian auto component industry. Measuring Business Excellence, v. 14, n. 2, p. 46-53, 2010. crossref

SORIANO-MEIER, H.; FORRESTER, P. L. A model for evaluating the degree of leanness of manufacturing firms. Integrated Manufacturing Systems, v. 13, n. 2, p. 104-109, 2002. crossref 
SRINIVASARAGHAVAN, J.; ALLADA, V. Application of Mahalanobis distance as a lean assessment metric. International Journal of Advanced Manufacturing Technology, v. 29, n. 11, p. 1159-68, 2006. crossref

TAJ, S. Applying lean assessment tools in Chinese hi-tech industries. Management Decision, v. 43, n. 4, p. 628-643, 2005. cross ref

TAJ, S. Lean manufacturing performance in China: assessment of 65 manufacturing plants. Journal of Manufacturing Technology Management, v. 19, n. 2, p. 217-234, 2008. VIMAL, K. E. K.; VINODH, S. Leanness evaluation using IF-THEN rules. International Journal of Advanced Manufacturing Technology, v. 63, n. 1-4, p. 407-413, 2012.

crossref

VIMAL, S.; VINODH, S. Application of artificial neural network for fuzzy logic based leanness assessment. Journal of Manufacturing Technology Management, v. 24, n. 2, p. 274-292, 2013. crossref

VINODH, S.; CHINTHA, S. K. Leanness assessment using multi-grade fuzzy approach. International Journal of Production Research, v. 49, n. 2, p. 431-445, 2011. crossref

VINODH, S.; KUMAR, C. D. Development of computerized decision support system for leanness assessment using multi grade fuzzy approach. Journal of Manufacturing Technology Management, v. 23, n. 4, p. 503-516, 2012. crossref

VINODH, S.; PRAKASH, N. H.; SELVAN, K. E. Evaluation of leanness using fuzzy association rules mining. International Journal of Advanced Manufacturing Technology, v. 57, n. 1-4, p. 343-352, 2011. crossref

VINODH, S.; VIMAL, K. E. K. Thirty criteria based leanness assessment using fuzzy logic approach. International Journal of Advanced Manufacturing Technology, v. 60, n. 9-12, p. 1185-1195, 2012. crossref

WALTER, O. M. F. C.; TUBINO, D. F. Métodos de avaliação da implantação da manufatura enxuta: uma revisão da literatura e classificação. Gestão \& Produção, v. 20, n. 1, p. 23-45, 2013. cross ref

WAN, H. D.; CHEN, F. F. A leanness measure of manufacturing systems for quantifying impacts of lean initiatives. International Journal of Production Research, v. 46, n. 23, p. 6567-6584, 2008. cross ref

WAN, H. D.; CHEN, F. F. Decision support for lean practitioners: a web-based adaptive assessment approach. Computers in Industry, v. 60, n. 4, p. 277-283, 2009. crossref

WEBSTER, J.; WATSON, R. T. Analyzing the past to prepare for the future: writing a literature review. MIS Quarterly, v. 26, n. 2, p. 8-23, 2002.

WOMACK, J. P.; JONES, D. T.; ROOS, D. A máquina que mudou o mundo. Rio de Janeiro: Campus, 1992.

\section{Dados dos autores:}

\section{Nome completo: Flora Magna do Monte Vilar}

Filiação institucional: Universidade Federal da Paraíba

Departamento: Engenharia de Produção

Função ou cargo ocupado: Mestranda

Endereço completo para correspondência: UFPB - Universidade Federal da Paraíba, Departamento de Engenharia de Produção, Centro de Tecnologia - Campus I, Bloco G, Cidade Universitária, João Pessoa - PB, Brasil - Cx. Postal: 5045 - CEP: 58.051-970

Telefones para contato: (83) 3216-7549

e-mail: floramontevilar@gmail.com 
Nome completo: Luciano Costa Santos

Filiação institucional: Universidade Federal da Paraíba

Endereço completo para correspondência: UFPB - Universidade Federal da Paraíba, Departamento de Engenharia de Produção, Centro de Tecnologia - Campus I, Bloco G, Cidade Universitária, João Pessoa - PB, Brasil - Cx. Postal: 5045 - CEP: 58.051-970

Telefones para contato: (83) 3216-7685

e-mail:luciano@ct.ufpb.br

\section{Nome completo: Cláudia Fabiana Gohr}

Filiação institucional: Universidade Federal da Paraíba

Departamento: Engenharia de Produção

Função ou cargo ocupado: Professora

Endereço completo para correspondência: UFPB - Universidade Federal da Paraíba, Departamento de Engenharia de Produção, Centro de Tecnologia - Campus I, Bloco G, Cidade Universitária, João Pessoa - PB, Brasil - Cx. Postal: 5045 - CEP: 58.051-970

Telefones para contato: (83) 3216-7549

e-mail: claudiagohr@ct.ufpb.br

Nome completo: Mateus Marcelino da Silva

Filiação institucional: Universidade Federal da Paraíba

Departamento: Engenharia de Produção

Função ou cargo ocupado: Estudante

Endereço completo para correspondência: UFPB - Universidade Federal da Paraíba, Departamento de Engenharia de Produção, Centro de Tecnologia - Campus I, Bloco G, Cidade Universitária, João Pessoa - PB, Brasil - Cx. Postal: 5045 - CEP: 58.051-970

Telefones para contato:

(83) 3216-7549

e-mail:mateusmarcelinos@hotmail.com

Submissão: 05/10/2015

Aceito: 05/03/2016 